\title{
Article \\ Electron-Impact Ionization of Heavy Atoms Using the Time-Dependent Close-Coupling Method
}

\author{
Michael S. Pindzola ${ }^{1, *}$, Stuart D. Loch ${ }^{1}$ and James P. Colgan ${ }^{2}$ \\ 1 Department of Physics, Auburn University, Auburn, AL 36849, USA; loch@physics.auburn.edu \\ 2 Theoretical Division, Los Alamos National Laboratory, Los Alamos, NM 87545, USA; jcolgan@lanl.gov \\ * Correspondence: pindzms@auburn.edu
}

check for updates

Citation: Pindzola, M.S.; Loch, S.D.; Colgan, J.P. Electron-Impact Ionization of Heavy Atoms Using the Time-Dependent Close-Coupling Method. Atoms 2021, 9, 32. https:// doi.org/10.3390/atoms9020032

Academic Editor: Jean-Christophe Pain

Received: 15 May 2021

Accepted: 2 June 2021

Published: 8 June 2021

Publisher's Note: MDPI stays neutral with regard to jurisdictional claims in published maps and institutional affiliations.

Copyright: (c) 2021 by the authors. Licensee MDPI, Basel, Switzerland. This article is an open access article distributed under the terms and conditions of the Creative Commons Attribution (CC BY) license (https:// creativecommons.org/licenses/by/ $4.0 /)$.

\begin{abstract}
The time-dependent close-coupling method has been recently applied to calculate electronimpact direct ionization cross sections for the $\mathrm{Kr}, \mathrm{W}$, and $\mathrm{Pb}$ atoms. An overview is presented for these three heavy neutral atom systems. When the direct ionization cross sections are combined with excitation-autoionization cross sections, the total ionization cross sections were found to be in reasonable agreement with crossed-beams measurements for $\mathrm{Kr}$ and $\mathrm{Pb}$.
\end{abstract}

Keywords: ionization; atoms; electron

\section{Introduction}

Electron-impact direct ionization cross sections for the $\mathrm{Kr}, \mathrm{W}$, and $\mathrm{Pb}$ atoms have recently been calculated using the time-dependent close-coupling (TDCC) method [1-3]. These elements have a number of important applications, with $\mathrm{W}$ being used as a plasma facing component on fusion energy experiments [4], Pb being an element with one of the highest cosmic abundances for heavy species and being made in neutron star merges [5], and $\mathrm{Kr}$ is of use in the radiative cooling of edge plasmas in fusion devices [6] and in Hall thrusters [7]. When the TDCC direct ionization cross sections for $\mathrm{Kr}$ [1] are combined with distorted-wave excitation-autoionization cross sections, the total ionization cross sections are found to be in reasonable agreement with crossed-beams measurements [8]. In addition, the TDCC direct ionization cross sections for $\mathrm{Pb}$ [3] are found to be in good agreement with converged close-coupling direct ionization cross sections [9]. When the direct ionization cross sections for $\mathrm{Pb}$ are combined with R-matrix B-spline excitation-autoionization cross sections [9], the total ionization cross sections are found to be in reasonable agreement with crossed-beams measurements [10,11]. For both $\mathrm{Kr}$ and $\mathrm{Pb}$, the non-perturbative TDCC results for direct ionization were found to be significantly lower than perturbative distorted-wave cross sections. There are currently no experimental measurements for the electron-impact ionization of the $\mathrm{W}$ atom. Non-perturbative ionization calculations for neutral heavy elements are challenging, thus the work presented here is intended as a review of recent TDCC results for such systems.

The rest of the paper is structured as follows: in Section 2 we review the timedependent close-coupling method for the electron ionization of atoms, in Section 3 we present TDCC electron ionization cross sections for $\mathrm{Kr}, \mathrm{W}$, and $\mathrm{Pb}$, and in Section 4 we give a brief summary. Unless otherwise stated, all quantities are given in atomic units.

\section{Theory}

The total single ionization cross section is given by:

$$
\sigma_{\text {total }}=\sum_{n_{0} l_{0}} \sigma_{\text {ion }}\left(n_{0} l_{0}\right)+\sum_{n_{1} l_{1} \rightarrow n_{2} l_{2}} \sigma_{\text {exc }}\left(n_{1} l_{1} \rightarrow n_{2} l_{2}\right) B\left(n_{1} l_{1} \rightarrow n_{2} l_{2}\right),
$$

where $\sigma_{\text {ion }}\left(n_{0} l_{0}\right)$ is the direct ionization cross section for all $n_{0} l_{0}$ subshells of the initial configuration for which the ionization potential is less than the double ionization potential, 
$\sigma_{\text {exc }}\left(n_{1} l_{1} \rightarrow n_{2} l_{2}\right)$ is the excitation cross section to an autoionizing configuration, and $B\left(n_{1} l_{1} \rightarrow n_{2} l_{2}\right)$ is a branching ratio for autoionization leading to single ionization. The double ionization potential is for the whole atom.

The TDCC equations for each $L S$ symmetry are given by:

$$
\begin{aligned}
c c c i \frac{\partial P_{l_{1} l_{2}}^{L S}\left(r_{1}, r_{2}, t\right)}{\partial t}= & \left(T_{l_{1}}\left(r_{1}\right)+T_{l_{2}}\left(r_{2}\right)\right) P_{l_{1} l_{2}}^{L S}\left(r_{1}, r_{2}, t\right) \\
& +\sum_{l_{1}^{\prime}, l_{2}^{\prime}} V_{l_{1} l_{2}, l_{1}^{\prime} l_{2}^{\prime}}^{L}\left(r_{1}, r_{2}\right) P_{l_{1}^{\prime} l_{2}^{\prime}}^{L S}\left(r_{1}, r_{2}, t\right),
\end{aligned}
$$

where

$$
T_{l_{i}}\left(r_{i}\right)=-\frac{1}{2} \frac{\partial^{2}}{\partial r_{i}^{2}}+\frac{l_{i}\left(l_{i}+1\right)}{2 r_{i}^{2}}-\frac{Z}{r_{i}}+V_{H}\left(r_{i}\right)+V_{X}\left(r_{i}\right),
$$

$V_{H}\left(r_{i}\right)$ is the direct Hartree potential, $V_{X}\left(r_{i}\right)$ is a local exchange potential, and $V_{l_{1} l_{2}, l_{1}^{\prime} l_{2}^{\prime}}^{L}\left(r_{1}, r_{2}\right)$ is the electrostatic repulsion operator. Details on the potentials are found in the publications for $\mathrm{Kr}$ [4], $\mathrm{W}$ [5], and $\mathrm{Pb}[6]$.

At an appropriate time $t=T$ after the collision, the direct ionization probability is given by:

$$
\begin{aligned}
c c P_{i o n}^{L S}= & -\sum_{n, l} \delta\left(l_{1} l L\right) \int_{0}^{\infty} d r_{1}\left|\int_{0}^{\infty} d r_{2} P_{l_{1} l}^{L S}\left(r_{1}, r_{2}, T\right) P_{n l}\left(r_{2}\right)\right|^{2} \\
& -\sum_{n, l} \delta\left(l l_{2} L\right) \int_{0}^{\infty} d r_{2}\left|\int_{0}^{\infty} d r_{1} P_{l l_{2}}^{L S}\left(r_{1}, r_{2}, T\right) P_{n l}\left(r_{1}\right)\right|^{2} \\
& +\sum_{n, l} \sum_{n^{\prime}, l^{\prime}} \delta\left(l l^{\prime} L\right)\left|\int_{0}^{\infty} d r_{1} \int_{0}^{\infty} d r_{2} P_{l l^{\prime}}^{L S}\left(r_{1}, r_{2}, T\right) P_{n l}\left(r_{1}\right) P_{n^{\prime} l^{\prime}}\left(r_{2}\right)\right|^{2},
\end{aligned}
$$

where $P_{l_{1} l_{2}}^{L S}\left(r_{1}, r_{2}, t\right)$ are solutions to the TDCC equations, $P_{n l}(r)$ are bound radial orbitals for the valence electron, and $\delta\left(l_{1} l_{2} l_{3}\right)$ is an algebraic triangle relation. The appropriate time is found when the scattered electrons are far enough apart so that their interaction is small.

The TDCC direct ionization cross section is given by:

$$
\sigma_{\text {ion }}\left(n_{0} l_{0}\right)=\frac{\pi w_{0}}{8\left(2 l_{0}+1\right) E} \sum_{L, S}(2 L+1)(2 S+1) P_{\text {ion }}^{L S},
$$

where $w_{0}$ is the occupation number of the initial subshell with angular momentum $l_{0}$ and $E=\frac{k_{1}^{2}}{2}+\frac{k_{2}^{2}}{2}$.

\section{Results}

Although there are many calculations for the direct ionization of the $\mathrm{Kr}, \mathrm{W}$, and $\mathrm{Pb}$ atoms, for example the calculation of Goswarni et al. for W [12], we compare the TDCC and distorted-wave calculations with only the very accurate convergent close-coupling calculations for $\mathrm{Pb}$ [9]. For most atoms the inclusion of the free particle correlation effects found in the TDCC method is found to lower the cross section in comparison to distortedwave cross sections that do not include free particle correlation effects. We note that direct impact double ionization cross sections are generally much smaller than direct impact single ionization cross sections and appear at higher electron energies.

The TDCC calculations for the direct ionization of the $4 p$ and $4 s$ subshells of $\mathrm{Kr}$ were carried out on a $720 \times 720$ point $\left(r_{1}, r_{2}\right)$ variable mesh lattice [1]. A simple analytic formula was used to smoothly join the calculated TDCC cross sections given by the blue squares. As shown in Figures 1 and 2 the TDCC cross sections were found to be substantially lower than distorted-wave cross sections as has been observed for other neutral systems. Total ionization cross sections for $\mathrm{Kr}$ are shown in Figure 3, in which theory was compared to crossed-beams measurements [8]. Both the TDCC and distorted-wave direct ionization 
cross sections for the $4 p$ and $4 s$ subshells were added to rather small $4 s \rightarrow n l$ excitationautoionization cross sections calculated using the distorted-wave method. The theoretical results are slightly higher than the measurements, but significantly improved over the perturbative results.

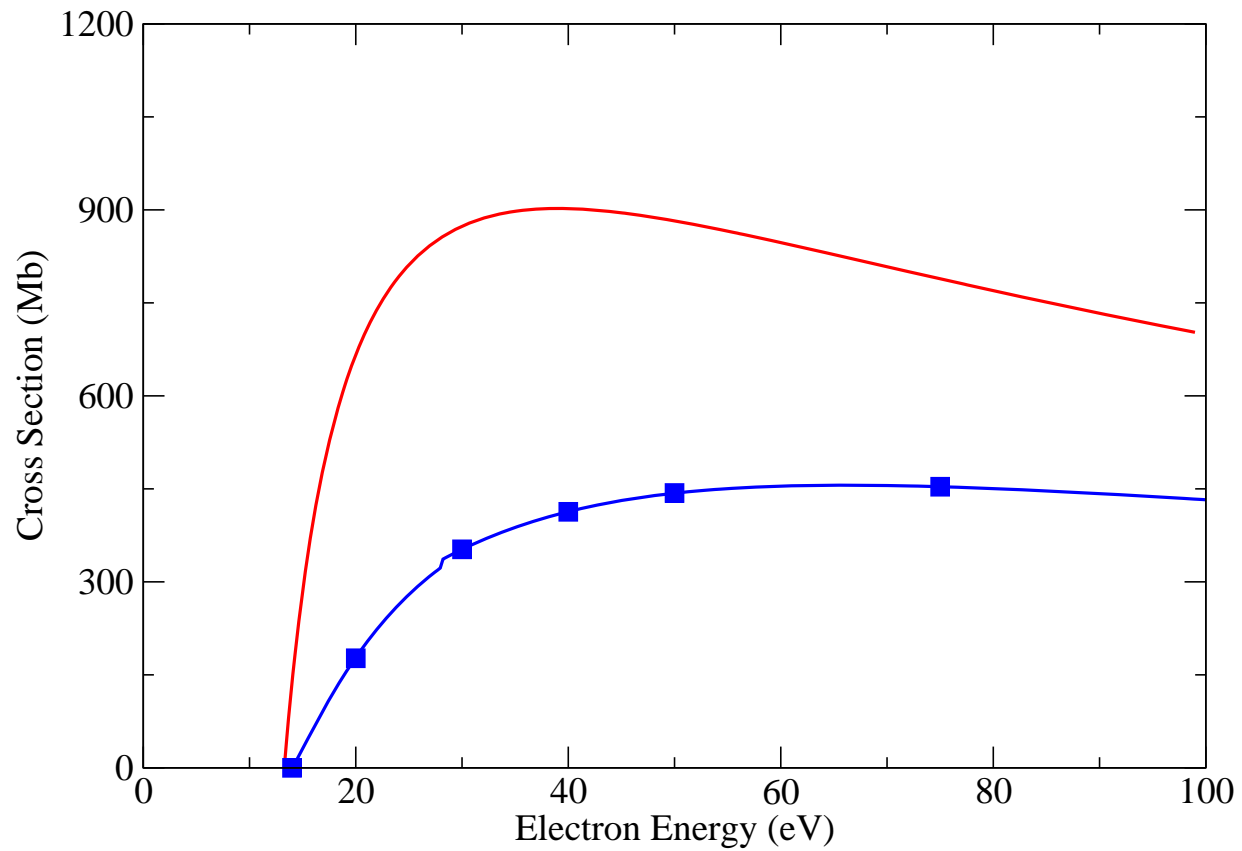

Figure 1. Electron ionization of the $4 p$ subshell of $\mathrm{Kr}$ Solid line (red): distorted-wave method, solid line with squares (blue): time-dependent close-coupling method $\left(1.0 \mathrm{Mb}=1.0 \times 10^{-18} \mathrm{~cm}^{2}\right)$.

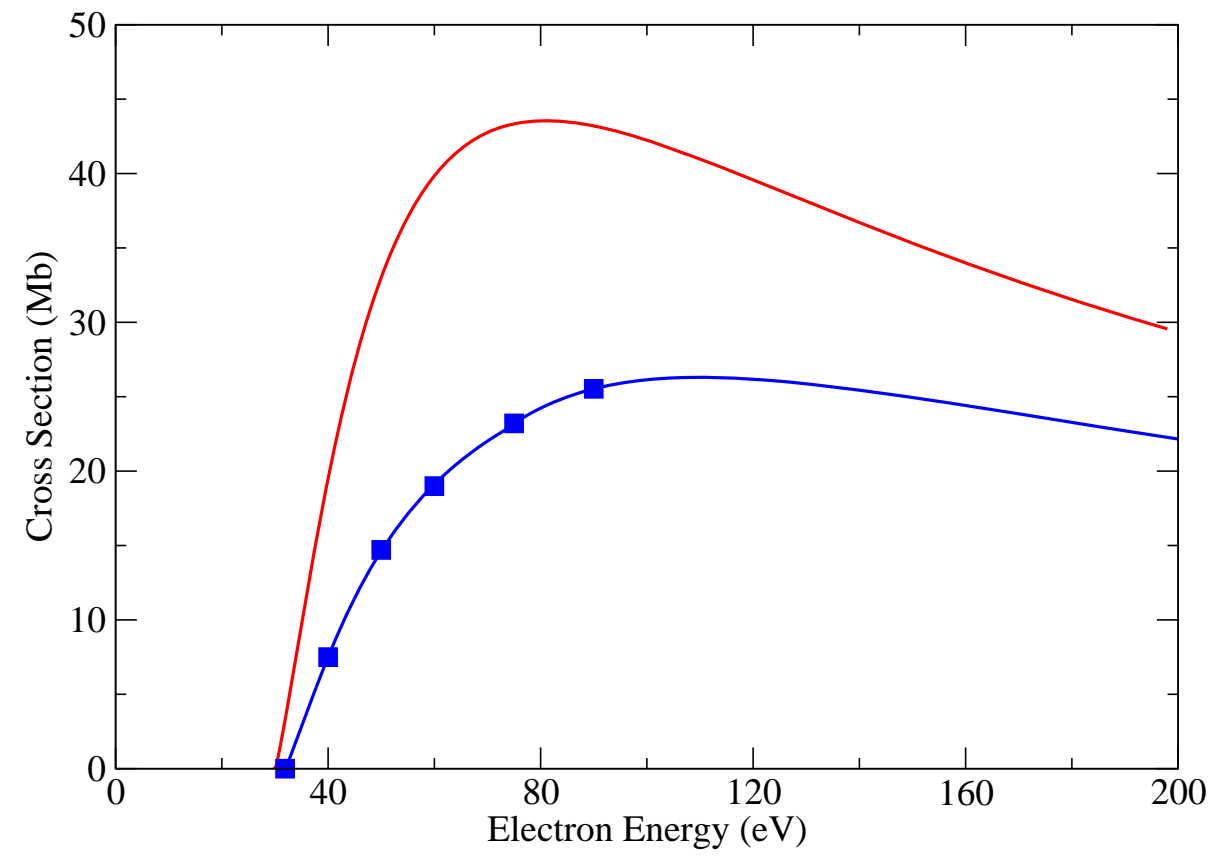

Figure 2. Electron ionization of the $4 s$ subshell of $\mathrm{Kr}$ Solid line (red): distorted-wave method, solid line with squares (blue): time-dependent close-coupling method $\left(1.0 \mathrm{Mb}=1.0 \times 10^{-18} \mathrm{~cm}^{2}\right)$. 




Figure 3. Electron-impact ionization of Kr. Dashed line (red): direct ionization of the $4 p$ and $4 s$ subshells using the CADW method, solid line (red): direct ionization of the $4 p$ and $4 s$ subshells using the CADW method plus all $4 s \rightarrow n l$ excitations, dashed line with squares (blue): Direct ionization of the $4 p$ and $4 s$ subshells using the TDCC method, solid line (blue): direct ionization of the $4 p$ and $4 s$ subshells using the TDCC method plus all $4 s \rightarrow n l$ excitations, circles (green): crossed-beams measurements [8], $\left(1.0 \mathrm{Mb}=1.0 \times 10^{-18} \mathrm{~cm}^{2}\right)$.

The TDCC calculations for the direct ionization of the $6 s$ and $5 d$ subshells of W were carried out on a $480 \times 480$ point $\left(r_{1}, r_{2}\right)$ fixed mesh lattice [2]. As shown in Figure 4 the TDCC cross sections for the $6 s$ subshell were found to be slightly lower than semirelativistic and fully-relativistic distorted-wave cross sections. However, as shown in Figure 5, the TDCC cross sections for the $5 d$ subshell were found to be substantially lower than semi-relativistic and fully-relativistic distorted-wave cross sections.

The TDCC calculations for the direct ionization of the $6 p$ and $6 s$ subshells of $\mathrm{Pb}$ were carried out on a $1344 \times 1344$ point $\left(r_{1}, r_{2}\right)$ variable mesh lattice [3]. As shown in Figure 6, the TDCC cross sections for the $6 p$ subshell were found to be substantially lower than distorted-wave cross sections, but in good agreement with convergent close-coupling cross sections [9]. As shown in Figure 7, the TDCC cross sections for the $6 s$ subshell were found to be slightly lower than distorted-wave cross sections and slightly above convergent closecoupling cross sections [9]. We note that the $6 s$ subshell cross section is almost five times smaller than the $6 p$ subshell cross section with less agreement between the TDCC and convergent close-coupling cross sections. Total ionization cross sections for $\mathrm{Pb}$ are shown in Figure 8, in which theory was compared to crossed-beams measurements [10,11]. Both the TDCC and convergent close-coupling [9] direct ionization cross sections fall below the crossed-beams measurements $[10,11]$, while the convergent close-coupling calculation for direct ionization ionization added to R-matrix B-spline excitation-autoionization cross sections are in excellent agreement with experiments up to an incident energy of $20 \mathrm{eV}$. We note that the TDCC, distorted-wave, and convergent close-coupling calculations for the direct ionization cross sections all included a polarization potential. 




Figure 4. Electron-impact direct ionization of $\mathrm{W}$ from the 6 s subshell. Solid line (red): semi-relativistic distorted-wave cross section, dashed line (green): fully-relativistic distorted-wave cross section, dotdashed line with solid squares (blue): TDCC cross section $\left(1.0 \mathrm{Mb}=1.0 \times 10^{-18} \mathrm{~cm}^{2}\right)$.

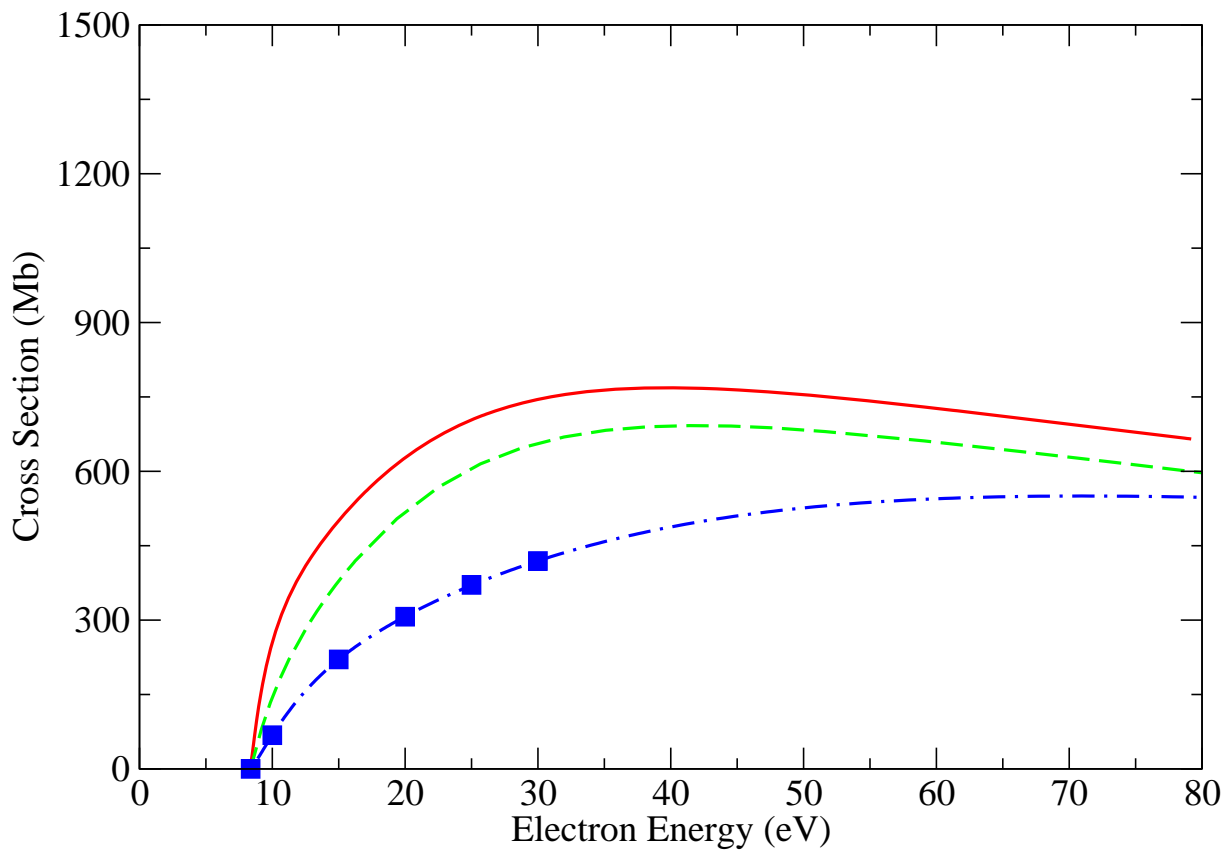

Figure 5. Electron-impact direct ionization of $\mathrm{W}$ from the $5 d$ subshell. Solid line (red): semi-relativistic distorted-wave cross section, dashed line (green): fully-relativistic distorted-wave cross section, dotdashed line with solid squares (blue): TDCC cross section $\left(1.0 \mathrm{Mb}=1.0 \times 10^{-18} \mathrm{~cm}^{2}\right)$. 


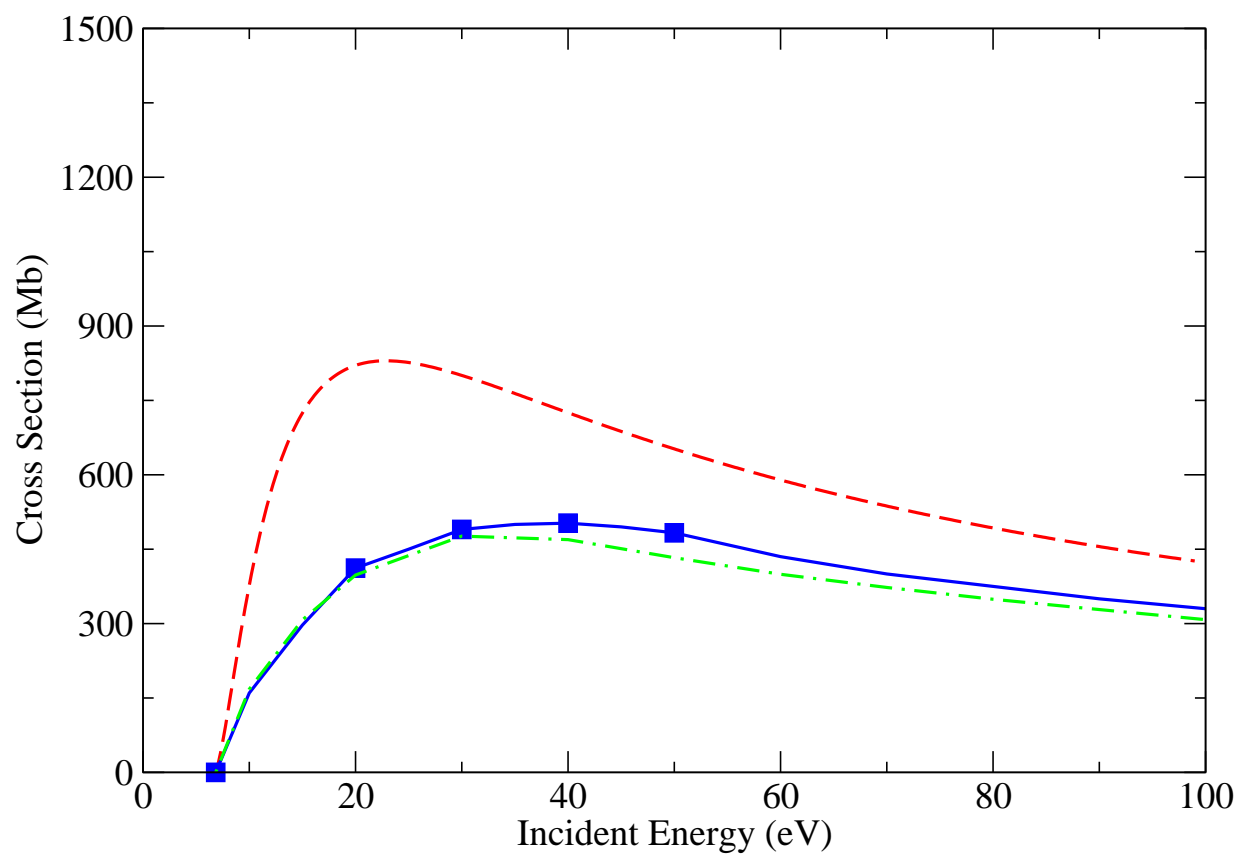

Figure 6. Electron-impact direct ionization of the $6 p$ subshell of $\mathrm{Pb}$. Dashed line (red): distorted-wave method with a polarization potential, solid line with squares (blue): time-dependent close-coupling method with a polarization potential, dot-dash line (green): convergent close-coupling method [9] with a polarization potential $\left(1.0 \mathrm{Mb}=1.0 \times 10^{-18} \mathrm{~cm}^{2}\right)$.

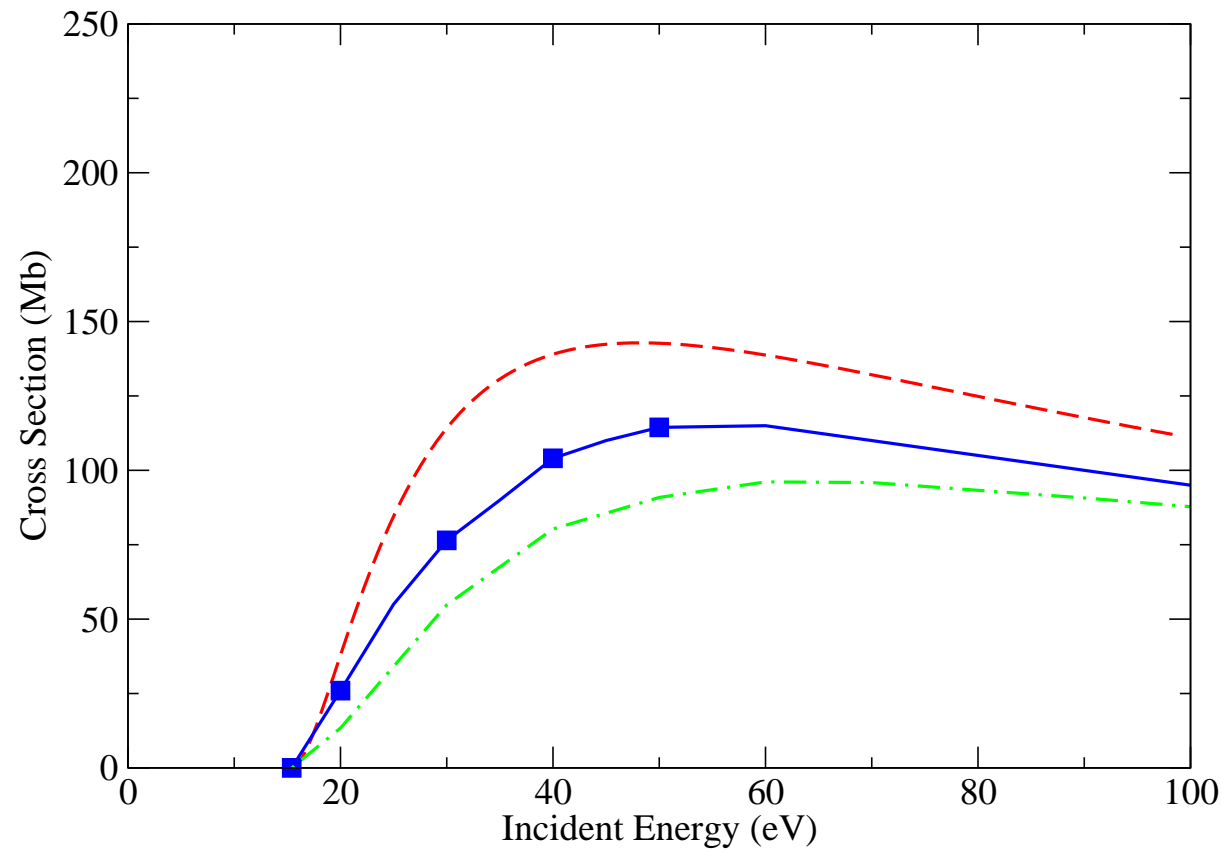

Figure 7. Electron-impact direct ionization of the $6 \mathrm{~s}$ subshell of $\mathrm{Pb}$. Dashed line (red): distorted-wave method with a polarization potential, solid line with squares (blue): time-dependent close-coupling method with a polarization potential, dot-dash line (green): convergent close-coupling method [9] with a polarization potential $\left(1.0 \mathrm{Mb}=1.0 \times 10^{-18} \mathrm{~cm}^{2}\right)$. 


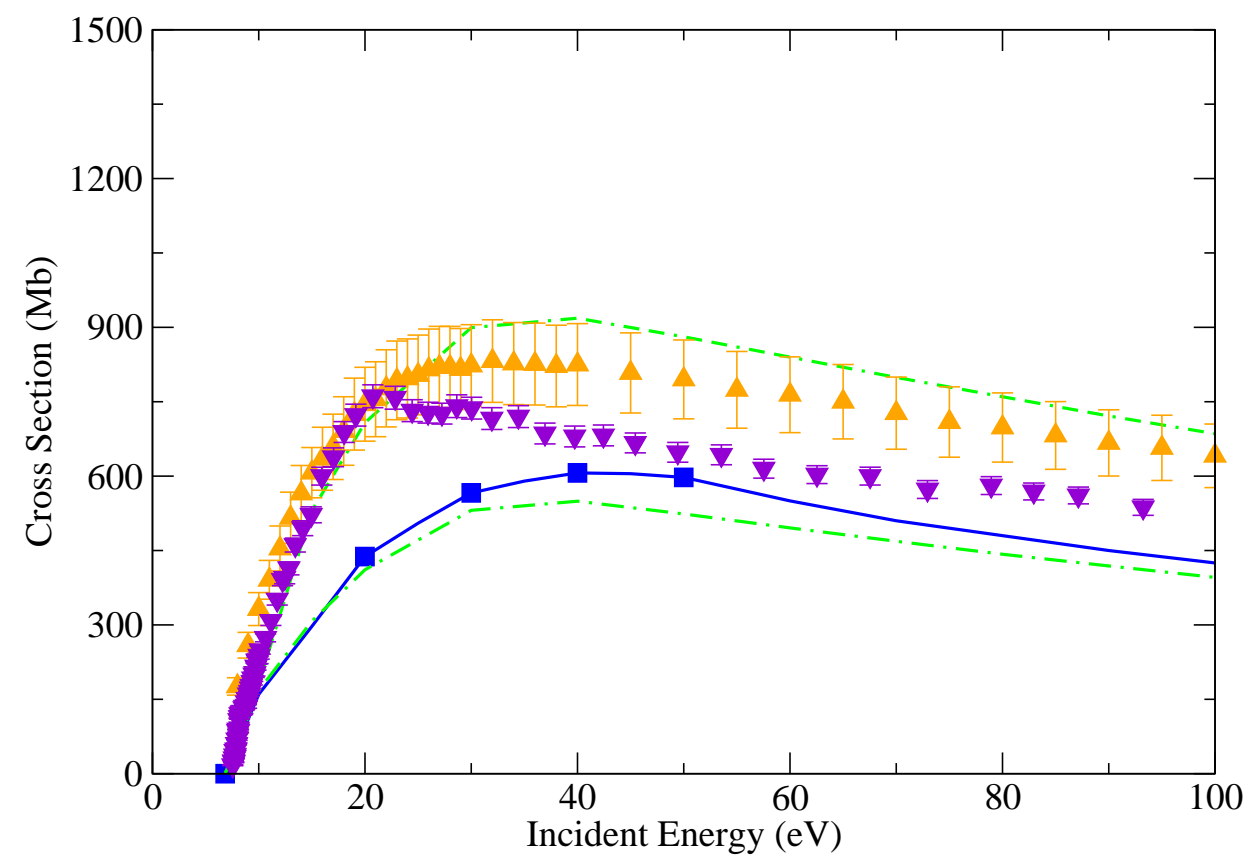

Figure 8. Electron-impact ionization of $\mathrm{Pb}$. Solid line with squares (blue): total TDCC direct ionization cross section with a polarization potential, dot-dash line (green): total CCC direct ionization cross section [9] with a polarization potential, dot-dash-dash line (green): total CCC direct ionization cross section with a polarization potential plus R-matrix B-spline indirect ionization cross section [9], up triangles (orange): crossed-beams measurements [10]. down triangles (violet): crossed-beams measurements [11] $\left(1.0 \mathrm{Mb}=1.0 \times 10^{-18} \mathrm{~cm}^{2}\right)$.

\section{Summary}

In conclusion we have presented previous results for the electron-impact direct ionization of the $\mathrm{Kr}, \mathrm{W}$, and $\mathrm{Pb}$ atoms using the time-dependent close-coupling method. In the future we plan to calculate the direct ionization of the outer subshells of the $\mathrm{Ta}, \mathrm{W}, \mathrm{Au}$, and $\mathrm{U}$ atoms using the TDCC method with a polarization potential. We note that previous calculations for the direct ionization of the inner subshells of the $\mathrm{Au}$ and $\mathrm{U}$ atoms $[13,14]$ were made using a fully-relativistic distorted-wave method.

Author Contributions: Investigation, J.P.C.; Methodology, M.S.P.; Validation, S.D.L. All authors have read and agreed to the published version of the manuscript.

Funding: This research received no external funding.

Data Availability Statement: Not applicable.

Acknowledgments: This work was supported in part by grants from the US National Science Foundation, the US National Aeronautics and Space Administration, and the US Department of Energy. Computational work was carried out at the National Energy Research Scientific Computing Center (NERSC) in Berkeley, California and at the High Performance Computing Center (HLRS) in Stuttgart, Germany.

Conflicts of Interest: The authors declare no conflict of interest.

\section{References}

1. Pindzola, M.S.; Loch, S.D. Electron-impact ionization of the Kr atom. J. Phys. B 2019, 52, 245205. [CrossRef]

2. Pindzola, M.S.; Loch, S.D.; Foster, A.R. Electron-impact single and double ionization of W. J. Phys. B 2017, 50, 095201. [CrossRef]

3. Pindzola, M.S.; Loch, S.D.; Colgan, J.P. Electron-impact ionization of the Pb atom. Eur. Phys. J. D 2021, 75, 45.

4. Matthews, G.F.; Su, M.-G.; Sun, D.-X.; Fu, Y.-B.; Dong, C.-Z. Theoretical Analysis of $4 \mathrm{f}$ and 5p Inner-Shell Excitations of W-W ${ }^{3+}$ Ions. Phys. Scr. 2007, 128, 137. [CrossRef]

5. Alonso-Medina, A. Experimental determination of the Stark widths of $\mathrm{Pb}$ I spectral lines in a laser-induced plasma. Spectrochim Acta Port B 2008, 63, 598-602. [CrossRef] 
6. Kallenbach, A.; Bernert, M.; Dux, R.; Casali, L.; Eich, T.; Giannone, L.; Herrmann, A.; McDermott, R.; Mlynek, A.; Müller, H.W. Impurity seeding for tokamak power exhaust: From present devices via ITER to DEMO. Plasma Phys. Control. Fusion 2013, 55, 124041. [CrossRef]

7. Linnell, J.A.; Gillimore, A.D. Internal plasma potential measurements of a Hall thruster using plasma lens focusing. Phys. Plasmas 2006, 13, 103504. [CrossRef]

8. Syage, J.A. Electron-impact cross sections for multiple ionization of Kr and Xe. Phys. Rev. A 1992, 46, 5666. [CrossRef] [PubMed]

9. van Eck, M.P.; Fursa, D.V.; Bray, I.; Zatsarinny, O.; Bartschat, K. Cross sections for electronscattering from atomic lead. J. Phys. B 2020, 53, 015204. [CrossRef]

10. Freund, R.S.; Wetzel, R.C.; Shul, R.J.; Hayes, T.R. Cross-section measurements for electron-impact ionization of atoms. Phys. Rev. A 1990, 41, 3575. [CrossRef]

11. McCartney, P.C.E.; Shah, M.B.; Geddes, J.; Gilbody, H.B. Cross-section measurements for electron-impact ionization of atoms. J. Phys. B 1998, 31, 4821. [CrossRef]

12. Goswarni, B.; Naghma, R.; Antony, B. Calculation of electron impact total ionization cross sections for tungsten, uranium and their oxide radicals. Int. J. Mass Spectrom. 2014, 372, 8.

13. Pindzola, M.S. Electron-impact ionization of the inner subshells of Au. J. Phys. B 2015, 48, 015201. [CrossRef]

14. Pindzola, M.S. Electron-impact ionization of the inner subshells of uranium. Phys. Rev. A 2014, 90, 022708. [CrossRef] 